\title{
SYSTEM ANALYSIS OF PREVENTION OF MAN-MADE EMERGENCY SITUATIONS IN CONSEQUENCE OF FIRE IN THE PREMISES OF THE OBJECT
}

\author{
Ruslan Meleshchenko ${ }^{1}$ \\ ${ }^{1}$ Department of Fire and Rescue Training, National University of Civil Defence of Ukraine, Kharkiv, Ukraine \\ mel@nuczu.edu.ua \\ ORCID: http://orcid.org/0000-0001-5411-2030
}

ARTICLE INFO

Article history:

Received date 14.01.2020

Accepted date 26.01.2020

Published date 31.01.2020

Section:

Distance, Open \& Online Education

DO I

10.21303/2313-8416.2020.001150

KEYWORDS

emergency

fire in the premises

system analysis

prevention of emergency situations

gas environment

dynamics of the gas environment state

\section{A B S T RACT}

A systematic analysis of the occurrence of man-made emergencies due to a fire at the facilities is performed. It is established that in the event of fires, the gaseous medium in the premises of the facility serves as a means of transmitting hazardous effects to the facility. The object of influence may be maintenance personnel, technological equipment, units, the design of the room or the object itself. In this case, the state of the object of influence can be characterized by a certain scalar parameter, which in the general case represents the amount of loss (damage or risk) caused to the object by the influence of the danger object. It is shown that the scalar parameter is a certain functional of the states of the hazard object and the gas environment, characterizing the degree of emergency, numerically equal to the amount of losses caused to the object of exposure by the hazard object in the room. The possibility of preventing a man-made emergency due to a fire based on monitoring the parameters of the state of the gas environment in the premises of the facility is substantiated. It is noted that in real conditions there is no information about the indicated disturbances and the state of the hazard object. This is the main difficulty in reliably preventing a manmade emergency due to a fire in the premises of the facility. An experimental verification of the dynamics of the parameters of the state of the gaseous medium in the premises of an object during the combustion of alcohol, paper, wood and fabric in a model chamber is performed. The obtained data indicate that the real dynamics of the temperature, smoke, and $\mathrm{CO}$ content of the gaseous medium in the chamber upon ignition of the tested materials is not deterministic, but is clearly unsteady and non-linear with obvious signs of an unsteady trend and random disturbances. It is established that the nature of the random dynamics of the states of the gaseous medium at the time of ignition of materials changes dramatically. Moreover, these changes are of an unequal nature, depending on the type of combustible material. The obtained results indicate the possibility of preventing a man-made emergency due to a fire by means of operational monitoring of the parameters of the gas environment in the premises of the facility

(C) The Author(s) 2020. This is an open access article under the CC BY license http://creativecommons.org/licenses/by/4.0).

\section{Introduction}

An emergency usually occurs with the simultaneous presence of an object of danger (source of danger) and the object of its impact. In this case, the subject of the impact is significantly damaged [1], including the death of people and other material values, and also causes serious damage to the environment [2]. World statistics in recent years says that the annual number of man-made emergencies is three or more times the number of annual emergencies of a natural and other nature [3]. It is noted that the main sources of man-made emergencies are various potentially dangerous objects [4]. Following [5], in recent years there has been a steady tendency for an increase in emergencies due to a significant expansion of the types of hazards. The prerequisites for the emergence of hazards leading to emergencies in the man-made sphere are usually created by the person itself in the course of production activities. Therefore, human production activities should minimize the risk of such hazards that lead to the emergence and elimination of emergencies [6]. One of the ways to reduce the risk of possible hazards is considered to be measures to prevent emergencies. It is noted that the prevention of emergencies 
can reduce the cost of eliminating their consequences by 2-3 times [7], as well as significantly reduce or completely eliminate the loss of life. World statistics show that most of the manmade emergencies at the facilities are connected with fires in the premises, leading to the defeat and death of staff and significant destruction of technological equipment, facilities and the facilities themselves.

Given the existing variety of types of hazardous facilities, as well as the objective complexity of detecting fires at facilities, the prevention of an emergency of a man-made nature due to a fire in the facility's premises is relevant.

\section{Literature review}

The gas environment of the premises is a complex system with a dissipative structure, non-linear dynamics and self-organization properties. In the event of a fire in the room, the gaseous medium reacts accordingly to its occurrence. In such a system, classical methods are not able to detect emerging complex nonlinear relationships between its elements, since they are based on linear principles, which in this case are usually violated [8]. This leads to false ideas about the dynamics of the state of the gas environment in the premises in case of fire and does not allow on its basis to prevent emergencies due to fires at the facilities. However, to prevent emergencies, the nature of the dynamics of the state of the gas environment in the event of a fire is of paramount importance. Since, based on the analysis of the dynamics of the state of the gas environment at the beginning of a fire, it is possible to prevent the defeat and death of maintenance personnel, as well as the destruction of technological equipment and units in the premises of facilities [9]. It should be noted that at present, the methods of nonlinear dynamics of the state of complex systems are an active area of research in many disciplines $[10,11]$. In particular, in geophysics methods of time series analysis are used from the position of the theory of dynamical systems and fractal sets [12]. At the same time, methods for preventing man-made emergencies due to fire at facilities are not considered. The study of the features of the process of a fire in the premises based on experimental data is performed in [13].

However, the options for preventing man-made emergencies due to a fire in the premises are not considered. The influence of thermal radiation on the rate of heat generation in standard materials is evaluated in [14]. An experimental study of the combustion regimes of various materials under external thermal influence is performed in [15]. The study of the rate of heat release during a fire in typical rooms is the subject of [16]. It is noted that the dynamics of the state of the gas environment in the premises at the initial stage of the fire is complex, non-linear and non-stationary. The work [17] is devoted to increasing the speed of known methods for detecting fires under the indicated conditions. New methods for detecting fires by changing the state of the gaseous medium are not considered and the dynamics of the states of the gaseous medium are not studied. Methods of preventing emergencies due to fire based on the identification of changes in the conditions of the gas environment in the premises are not considered. Self-tuning methods for identifying fires are the subject of $[18,19]$. However, these methods are based on the averaged values of individual parameters of the state of the gaseous medium without taking into account the current dynamics of the parameters. Moreover, the results presented in [20] are limited to the analysis of only the dynamics of the adaptive threshold and the probability of detecting fires. The dynamics of the parameters of the state of the gaseous medium and their increments is not studied and is not considered. Techniques for preventing man-made emergencies due to fire based on increments in the state of the gas environment in the premises are not considered. The study of temporary covariance and mutual covariance of the main parameters of the state of the gaseous medium during fires in the room model was performed in [21]. It is noted that for detecting the onset of a fire in the premises, the current changes in the parameters of the state of the gaseous medium, rather than their average value, are more important. For example, methods are known that are suitable for identifying hazardous parameters of the state of a gaseous medium in a fire [22]. However, these methods are based on a stationary approach, which will allow revealing only averaged energy indicators of the parameters of the gaseous medium by lags and frequencies. Moreover, these methods do not take into account the time-frequency structure of the state parameters of the gaseous medium.

In [23], methods of time-frequency localization are considered, which turn out to be difficult to implement and unsuitable for the rapid detection of fire in the premises of the facility and 
the possibility of their use to prevent emergency situations. Due to the unsteady dynamics of the parameters of the state of the gaseous medium in rooms during a fire in [24], a method is proposed based on the application of the short-term Fourier transform to individual stationary fragments of the unsteady dynamics of parameters. However, at the initial stage of a fire in the room, it is not possible to isolate stationary fragments of the dynamics of the parameters of the gaseous medium state. In this case, the gaseous medium as a complex dynamic system that generates non-stationary hazardous state parameters during a fire is not considered and is not studied. An experimental study of the dynamics of the burning rate of various materials in closed and ventilated rooms is the subject of work [25]. However, it does not contain data on the structure of the interaction of hazardous parameters of the state of the gaseous medium during ignition of materials in the premises. The work [26] is devoted to the study of increments of certain hazardous parameters of the state of the gaseous medium for the detection of early fires in rooms. However, the results in it are limited to the analysis of traditional statistical indicators for increments of the state parameters of the gaseous medium. Moreover, the study of the structural features of the dynamics of the state parameters of the gaseous medium in a multidimensional phase space is not considered. In [21-26], it is noted that fires are not only a source of fire hazard, but also a source of violation of the parameters of the initial equilibrium state of the gaseous medium in the premises of the facility. In the general case, the state of the gaseous medium during fires in rooms is characterized by a more complex nonlinear dynamics of the main parameters.

The general methods of time-frequency identification of nonlinear dynamical systems are considered in $[27,28]$. The application of the method of short-term Fourier transform for the analysis of non-stationary parameters of processes is discussed in [29]. At the same time, the methods [27-29] turn out to be quite complicated, which does not allow using them for the prevention of man-made emergencies due to fires in the premises. Methods of analysis of the nonlinear dynamics of systems are not considered in [27-29]. Although for the early detection of fires and the prevention of man-made emergencies due to fires in the premises, first of all, a systematic analysis of the dynamics of states and state increments of the gas environment in the room is necessary, taking into account the corresponding dimensionality of the phase space. However, to date, such an analysis is not available in the known literature. For example, in [30], a time-frequency method is considered for analyzing the structural features of the dynamics of hazardous parameters of a gaseous medium at the stage of a fire in rooms. It is noted that this method is difficult to implement and is not operational. Moreover, the effectiveness of the method substantially depends on the parameters of the window functions used for averaging. In addition, this method, being an energy one, does not allow to study the fine structure of the dynamics of dangerous parameters of the state of the gaseous medium in the corresponding phase space.

Thus, due to the complex dynamics of hazardous parameters of the state of the gaseous medium during a fire in the premises, various time-frequency methods are used to identify them. However, these methods have several disadvantages: complexity, limited sensitivity and lack of responsiveness. Therefore, the application of these methods to prevent man-made emergencies due to fires in the premises of the facilities is problematic. Moreover, fractal methods of nonlinear dynamics should be considered more promising for the prevention of such emergencies [31]. However, their application for the prevention of man-made emergencies due to fires in the premises of objects, first of all, requires a systematic analysis of the prevention of these emergencies. Therefore, an important and unresolved part of the general problem is a system analysis of the prevention of man-made emergencies due to a fire in the premises of the facility.

\section{The aim and objectives of research}

The aim of research is a system analysis of the prevention of man-made emergencies due to a fire in the premises of the facility in the form of a system for the interaction of the hazard, the environment and the hazard.

To achieve the aim of research, the following objectives are set:

- perform a system analysis of the occurrence of man-made emergencies due to fire in the premises of the facility;

- substantiate the possibility of preventing a man-made emergency due to a fire by monitoring the parameters of the state of the gas environment in the premises of the facility; 
- verify experimentally the dynamics of the parameters of the state of the gaseous medium in the premises of the facility during the fire of alcohol, paper, wood and fabric in a model chamber simulating an unpressurized technological facility premises.

\section{System analysis of the occurrence of man-made emergencies due to a fire in the technolog- ical premises of the facility}

A system analysis of the occurrence of a man-made emergency due to fires in the premises of the facility is based on the representation of the premises as a system consisting of interacting objects. Let the facility's premises represent some system $S=O \bigcup E$ consisting of a hazard object $O$ (source of ignition and fire), which is the source of the considered emergencies, and some environment $E=C \bigcup R$, where $C$ is the gas environment of the room, and $R$ is an arbitrary object of influence of the hazard object (maintenance personnel, technological equipment, units and design of the room or the object itself). In this case, the state of the affected object will be determined by the corresponding sign (or signs) of emergencies.

In the emergency system under consideration, the hazard object $O$ affects the object exposed to hazard $\mathrm{R}$ through the gas environment of room $C$. In general, the hazard object $O$ is characterized by its own dangerous states $y \in O$. Such conditions can be, for example, fires of various materials or explosions of equipment and units in the room. This means that the states $y$ of the hazard object $\mathrm{O}$ at time instants $t \in[0, T]$ will be described by a random function $y=y(t)$.

In this case, the gas environment $C$ of the room transfers the influence of hazardous conditions from the source of danger to the object of its impact. In this case, the gas environment $C$ of the room is characterized by the corresponding state $z \in C$. In this case, the parameters of the state of the gas environment of the room may be, for example, hazardous to the operating personnel parameters of the gas environment. First of all, it is temperature, carbon monoxide concentration and smoke density. At various points in time, the state of the gaseous medium $C$ is characterized by a random function of time $z=z(t)$. In this case, the state of the object $R$, which is exposed to the hazard object $O$, can be characterized by some scalar parameter $l \in R$. In the general case, the parameter 1 represents the amount of loss (damage or risk) caused to the object R by the influence of the object $O$. In the case under consideration, the parameter 1 will be determined by the state of some united system $S G=O \bigcup C$ defined by the hazard object $O$ and the gas environment $C$. In this case, the parameter 1 will be some functional $l=l(y(t), z(t))$ characterizing the degree of emergency, numerically determined by the amount of loss (damage or risk) caused to the object of exposure $\mathrm{R}$ by the hazard object $\mathrm{O}$ in the room.

\section{Justification of the possibility of preventing a man-made emergency due to a fire by moni- toring the parameters of the state of the gas environment in the premises of the facility}

In order to justify the possibility of preventing a man-made emergency due to fires in the premises of the facility on the basis of monitoring the state of the gas environment, let's consider in more detail the loss (damage or risk) functional $l=l(y(t), z(t))$, which characterizes the degree of occurrence of a possible emergency. In the system of emergencies under consideration $S=O \bigcup E$, the state of the gaseous medium $z=z(t)$ in the room is determined by the state $y=y(t)$ of the hazard object $O$. This means that parameter 1 will be determined by the current state $z_{1}(t, y(t))$ of the gaseous medium in indoors, taking into account the current state $y(t)$ of the hazard object $O$, i. e., $l=l_{1}\left(z_{1}(t, y(t))\right)$. For example, if the damage caused to the object of influence R depends on the state $z_{1}=z_{1}(x, t, y(t))$ of dangerous parameters of the state of the gaseous medium at the point $\mathrm{x}$ of the object's space $\mathrm{x}$, then the parameter 1 can be represented as the corresponding one-dimensional integral function. In this case, the one-dimensional integral function will determine, for example, the inhalation dose of hazardous parameters of the state of the gaseous medium for maintenance personnel in the room. Thus, it is possible, on the basis of the current monitoring of the state $z_{1}=z_{1}(t, y(t))$ of hazardous parameters of the gas environment in the rooms where the technological equipment and maintenance personnel are located, to prevent an emergency of a man-made nature due to a fire in the facility's premises, taking into account losses caused to the object $R$ by the influence of the hazard object $O$. It should be noted that in real conditions the states $z_{1}=z_{1}(t, y(t))$ of the gas environment in the rooms depend not only on the state $y(t)$ of the hazard object (fire), but they are also subject to action of various disturbances. In most practical cases, data on these perturbations are absent and the only 
information for preventing emergencies is current measurements of the state of the gaseous medium, taking into account the corresponding perturbations [32]. This means that, in practice, instead of the state $z_{1}=z_{1}(t, y(t))$ considered above, the measurement of the state of the gaseous medium is available $\left.z_{1}=z_{1}\left(t, y\left(t, n_{i}(t)\right), n_{C}(t)\right)\right)$, where $n_{i}(t)$ and $n_{C}(t)$ determine the corresponding perturbations of the states of the hazard object and the gas environment of the room. In most cases, these perturbations are additive. In accordance with this, a system model of the process of detecting tanning in the premises of an object for assessing the state of the gaseous medium can be represented in the form shown in Fig. 1.

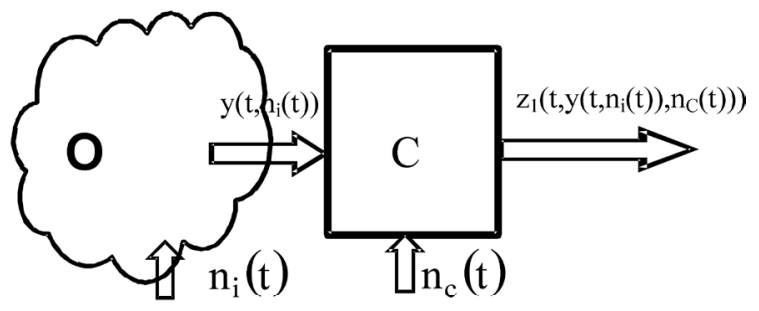

Fig. 1. System model of the process of detecting fire in the premises of the object

The condition $y\left(t, n_{i}(t)\right)$ of the hazard object is generally characterized by the uncertainty $\left(H_{1}, H_{0}\right)$ of the appearance or absence of sunburn, respectively, as well as by the influence of disturbances $n_{i}(t)$. Typically, disturbances $n_{i}(t)$ and $n_{c}(t)$ are caused by various interference at all stages of the danger and its transmission through the gas environment of the room. In the general case, following Fig. 1, the state $\left.z_{1}=z_{1}\left(t, y\left(t, n_{i}(t)\right), n_{C}(t)\right)\right)$ of the gaseous medium, taking into account the uncertainty of the occurrence of danger, can be represented in the operator form

$$
z_{1}(t)=C\left\{y\left(t, n_{i}(t)\right), n_{c}(t)\right\}_{H_{1}, H_{0}}
$$

where $C\{*\}_{H_{1}, H_{0}}$ - the operator of the hazard transmission medium $y(t)$ from the object (source), taking into account environmental disturbances $n_{c}(t)$ in the presence or absence of fire in the room. Taking into account (1), emergency prevention should be based on operational monitoring of the state $\left.z_{1}=z_{1}\left(t, y\left(t, n_{i}(t)\right), n_{c}(t)\right)\right)$ of the gaseous medium, which depends on the corresponding disturbances. There is no information on disturbances in real conditions. Therefore, the lack of information on perturbations and the dynamics of the parameters of the state of the gaseous medium in the absence and presence of ignition determine the main difficulty in reliably preventing an emergency of a man-made nature due to a fire in the premises.

\section{Experimental verification of the dynamics of the parameters of the gaseous medium state in the premises of the object when ignitions}

For real air-gas media, the transfer of the state effect $y(t)$ from the hazardous object through the medium is usually accompanied by various random disturbances. With this in mind, the transfer of dangerous states in the general case can be described by a generalized operator $C\left({ }^{*}\right)$ transforming states $y(t)$ into corresponding states $z_{1}(t)$ defined by (1). To verify the dynamics of state parameters (1), an experiment is conducted in accordance with the scheme shown in Fig. 2.

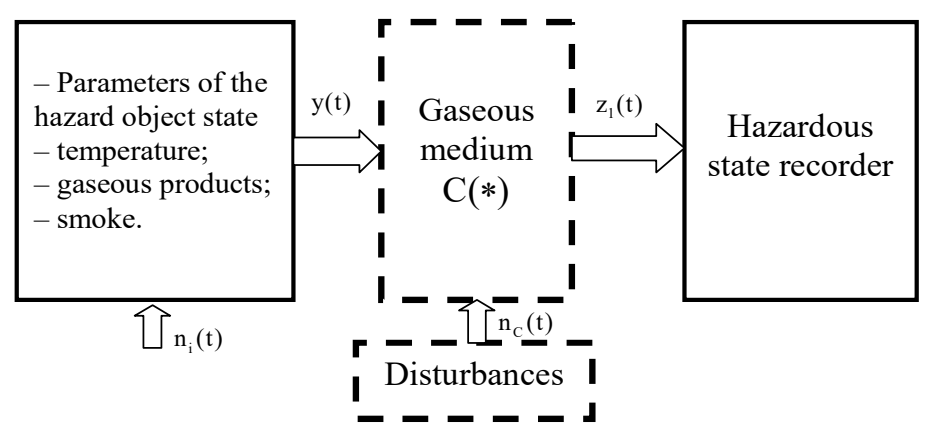

Fig. 2. The structural diagram of the experiment 
The experiment was carried out in an unpressurized chamber measuring $1020 \times 840 \times 520 \mathrm{~mm}$, in the upper part of which special sensors were placed to record the main hazardous parameters of the state of the gaseous medium when various test materials were ignited [33]. Alcohol $\left(\mathrm{C}_{2} \mathrm{H}_{5} \mathrm{OH}\right)$, fabric, paper, and wood (in the form of medium sized chips) were considered as combustible materials. The area of the test fire site was about $35 \mathrm{~cm}^{2}$, and the maximum flame height from the base of the hearth was about $200-230 \mathrm{~mm}$. The materials were ignited at approximately the $30 \mathrm{th}$ second (Fig. 3). During the experiment, the temperature of the gaseous medium, the density of the smoke, and the $\mathrm{CO}$ content in the chamber before and after the ignition of the materials were recorded for $300 \mathrm{~s}$.

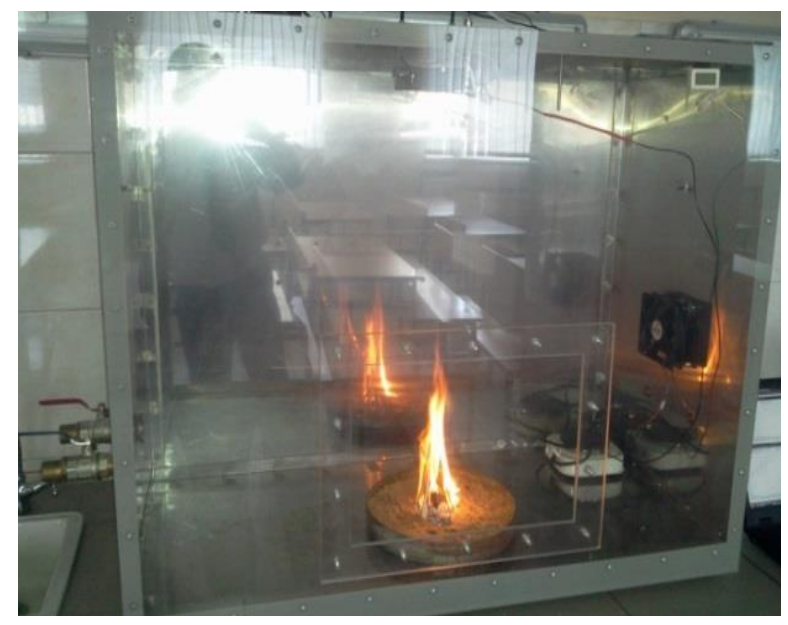

Fig. 3. Appearance of the chamber and the investigated source of material tanning

Fig. 4 presents the experimental results for the temperature of the gaseous medium in the chamber for various types of combustible material of the source (T1 - alcohol, T2 - paper, T3 wood, T4 - fabric).

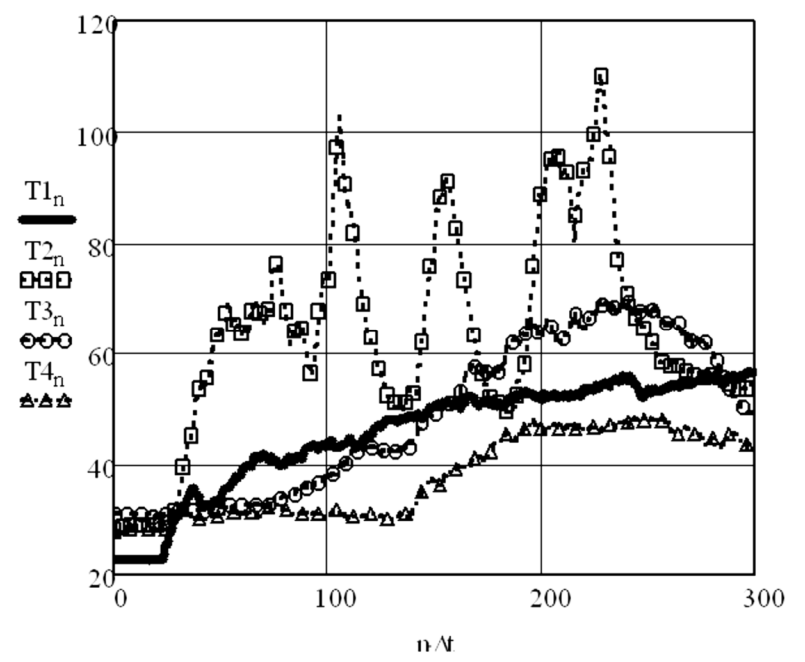

Fig. 4. The dynamics of the temperature of the gas environment of the chamber

Fig. 5, 6 show similar dependences, respectively, for smoke (D1 - alcohol, D2 - paper, D3 wood and D4 - fabric) and CO content (CO1 - alcohol, CO2 - paper, CO3 - wood and CO4 - fabric) of the gaseous medium in the chamber.

Dependencies in Fig. 4-6 illustrate the characteristic real dynamics of the main hazardous parameters of the state of the gaseous medium in the chamber during the ignition of the test materials under consideration, taking into account real perturbations and sensors that are used in modern heat, smoke and gas fire detectors. 


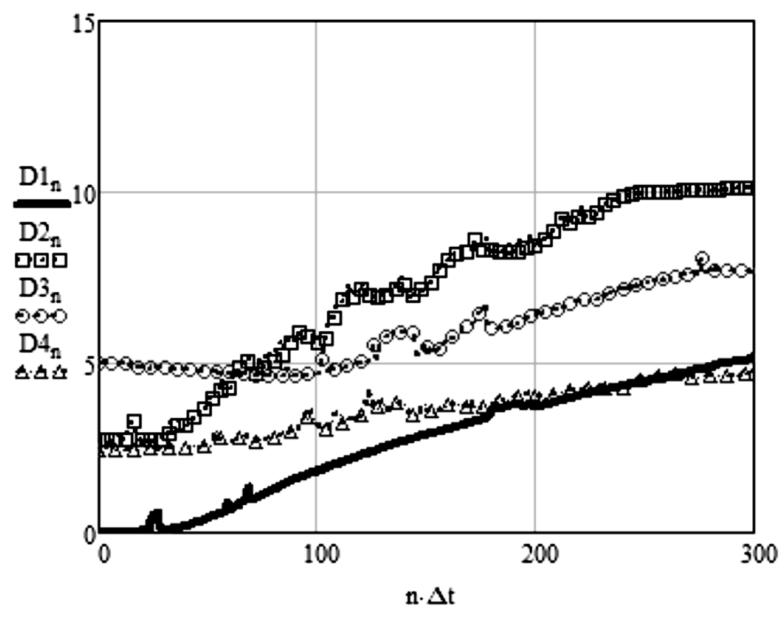

Fig. 5. The smoke dynamics of the gas environment of the chamber

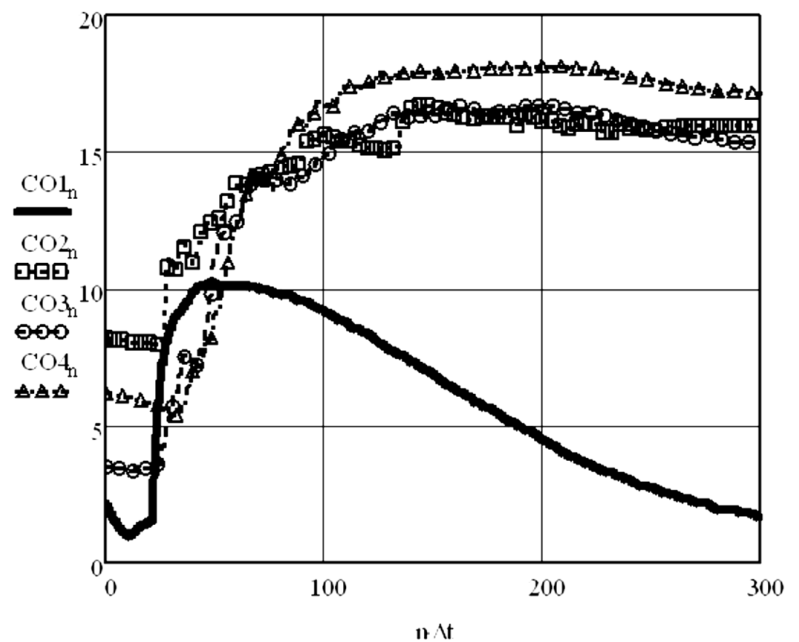

Fig. 6. Dynamics of $\mathrm{CO}$ content in the gaseous medium of the chamber

\section{Discussion of the results of an experimental verification of the dynamics of the parameters of the gaseous medium state in the chamber during ignition of materials}

Analysis of the data in Fig. 4-6 indicates that the real dynamics of the temperature, smoke, and $\mathrm{CO}$ content of the gaseous medium in the chamber upon ignition of the tested materials is not deterministic, but is clearly unsteady and non-linear with obvious signs of an unsteady trend and random disturbances. This proves the validity of the hypothesis about the random nature of the representation (1). From the analysis of the results it follows that the random dynamics of the state of the gaseous medium at the time of ignition of the materials changes dramatically. Moreover, these changes are of a different nature, which depends on the type of combustible material. This confirms that the operational monitoring of the state of the gas environment of the room allows to detect early tanning in the premises of the facility and to prevent an emergency of a man-made nature due to a fire in the premises of the facility.

The stochastic nature of the dynamics of the parameters of the state of the gas environment of the premises at the initial stage of ignition of materials requires the use of appropriate stochastic differential equations to describe it, which allow to solve a wide range of practical problems in reliably preventing man-made emergencies due to fire based on the state variable method. The use of stochastic differential equations in the form of equations of state allows to synthesize both optimal algorithms for recording the state parameters of the gaseous medium and technical devices for reliable warning of emergencies due to fires at an early stage of their occurrence in the premises of objects.

At the same time, methods for reliable prevention of man-made emergencies due to fires in facilities based on monitoring the dynamics of the parameters of the gas environment should take 
into account its nonlinear nature, as well as be invariant to the unsteady trend and random disturbances of the source and gas environment.

\section{Conclusions}

1. A system analysis of the occurrence of man-made emergencies due to a fire at the facilities has been performed. It is established that in the event of fires, the gaseous medium in the premises of the facility serves as a means of transmitting hazardous effects (temperature, carbon monoxide, and smoke) to the facility. The object of influence may be maintenance personnel, technological equipment, units, the design of the room or the object itself. In this case, the state of the object of influence can be characterized by some scalar parameter, which in the general case represents the amount of losses caused to the object by the influence of the object of danger. It is shown that the scalar parameter is a certain functional of the states of the hazard object and the gas environment, characterizing the degree of emergency, numerically equal to the amount of losses caused to the object of exposure by the hazard object in the room.

2. The possibility of preventing a man-made emergency due to a fire based on monitoring the parameters of the state of the gas environment in the premises of the facility in which the processing equipment is operated, as well as maintenance personnel, is justified. It is shown that emergency prevention should be based on operational monitoring of the state of the gaseous medium, which depends on the condition of the hazardous object, taking into account disturbances, as well as disturbances in the gas environment. It is noted that in real conditions there is no information about the indicated disturbances and the state of the hazard object. Therefore, the absence of this information is the main difficulty in providing reliable prevention of man-made emergencies due to fire in the premises of the facility.

3. An experimental check of the dynamics of the parameters of the state of the gaseous medium in the premises of the facility during the ignition of alcohol, paper, wood and fabric in a model chamber simulating an unpressurized technological room of the facility was performed. An analysis of the obtained data indicates that the real dynamics of the temperature, smoke, and $\mathrm{CO}$ content of the gas medium in the chamber upon ignition of the tested materials is not deterministic, but is non-stationary and non-linear with obvious signs of the presence of an unsteady trend and random disturbances. These changes are not the same character, which depends on the type of combustible material. At the same time, it is noted that methods for reliable prevention of manmade emergencies due to fires in facilities based on monitoring the dynamics of the parameters of the gas environment should take into account its non-linear nature, as well as be invariant to the unsteady trend and random disturbances of the source and gas environment. The obtained results indicate the possibility of preventing a man-made emergency due to a fire by means of operational monitoring of the parameters of the gas environment in the premises of the facility.

\section{References}

[1] Kovalov, A., Otrosh, Y., Ostroverkh, O., Hrushovinchuk, O., Savchenko, O. (2018). Fire resistance evaluation of reinforced concrete floors with fire-retardant coating by calculation and experimental method. E3S Web of Conferences, $60,00003$. doi: http://doi.org/10.1051/e3sconf/20186000003

[2] Semko, A. N., Beskrovnaya, M. V., Vinogradov, S. A., Hritsina, I. N., Yagudina, N. I. (2014). The usage of high speed impulse liquid jets for putting out gas blowouts. Journal of Theoretical and Applied Mechanics, 52 (3), 655-664.

[3] Kustov, M. V., Kalugin, V. D., Tutunik, V. V., Tarakhno, E. V. (2019). Physicochemical principles of the technology of modified pyrotechnic compositions to reduce the chemical pollution of the atmosphere. Voprosy khimii i khimicheskoi tekhnologii, 1, 92-99. doi: http://doi.org/10.32434/0321-4095-2019-122-1-92-99

[4] Semko, A., Rusanova, O., Kazak, O., Beskrovnaya, M., Vinogradov, S., Gricina, I. (2015). The use of pulsed high-speed liquid jet for putting out gas blow-out. The International Journal of Multiphysics, 9 (1), 9-20. doi: http://doi.org/10.1260/1750-9548.9.1.9

[5] Otrosh, Y., Kovalov, A., Semkiv, O., Rudeshko, I., Diven, V. (2018). Methodology remaining lifetime determination of the building structures. MATEC Web of Conferences, 230, 02023. doi: http://doi.org/10.1051/matecconf/201823002023

[6] Tiutiunyk, V. V., Ivanets, H. V., Tolkunov, I. A., Stetsyuk, E. I. (2018). System approach for readiness assessment units of civil defense to actions at emergency situations. Scientific Bulletin of National Mining University, 1, 99-105. doi: http://doi.org/ 10.29202/nvngu/2018-1/7

[7] Dubinin, D., Korytchenko, K., Lisnyak, A., Hrytsyna, I., Trigub, V. (2017). Numerical simulation of the creation of a fire fighting barrier using an explosion of a combustible charge. Eastern-European Journal of Enterprise Technologies, 6 (10 (90)), 11-16. doi: http://doi.org/10.15587/1729-4061.2017.114504 
[8] Vasiliev, M. I., Movchan, I. O., Koval, O. M. (2014). Diminishing of ecological risk via optimization of fire-extinguishing system projects in timber-yards. Scientific Bulletin of National Mining University, 5, 106-113.

[9] Kondratenko, O. M., Vambol, S. O., Strokov, O. P., Avramenko, A. M. (2015). Mathematical model of the efficiency of diesel particulate matter filter. Scientific Bulletin of National Mining University, 6, 55-61.

[10] Vasyukov, A., Loboichenko, V., Bushtec, S. (2016). Identification of bottled natural waters by using direct conductometry. Ecology, Environment and Conservation, 22 (3), 1171-1176.

[11] Pospelov, B., Andronov, V., Rybka, E., Meleshchenko, R., Borodych, P. (2018). Studying the recurrent diagrams of carbon monoxide concentration at early ignitions in premises. Eastern-European Journal of Enterprise Technologies, 3 (9 (93)), 34-40. doi: http://doi.org/10.15587/1729-4061.2018.133127

[12] Turcotte, D. L. (1997). Fractals and chaos in geology and geophysics. Cambridge university press. doi: http://doi.org/10.1017/ cbo9781139174695

[13] Poulsen, A., Jomaas, G. (2011). Experimental Study on the Burning Behavior of Pool Fires in Rooms with Different Wall Linings. Fire Technology, 48 (2), 419-439. doi: http://doi.org/10.1007/s10694-011-0230-0

[14] Zhang, D., Xue, W. (2010). Effect of heat radiation on combustion heat release rate of larch. Journal of West China Forestry Science, 39, 148.

[15] Ji, J., Yang, L., Fan, W. (2003). Experimental study on effects of burning behaviours of materials caused by external heat radiation. JCST, 9, 139.

[16] Peng, X., Liu, S., Lu, G. (2005). Experimental analysis on heat release rate of materials. Journal of Chongqing University, $28,122$.

[17] Andronov, V., Pospelov, B., Rybka, E. (2017). Development of a method to improve the performance speed of maximal fire detectors. Eastern-European Journal of Enterprise Technologies, 2 (9 (86)), 32-37. doi: http://doi.org/10.15587/17294061.2017.96694

[18] Pospelov, B., Andronov, V., Rybka, E., Skliarov, S. (2017). Design of fire detectors capable of self-adjusting by ignition. Eastern-European Journal of Enterprise Technologies, 4 (9 (88)), 53-59. doi: http://doi.org/10.15587/1729-4061.2017.108448

[19] Andronov, V., Pospelov, B., Rybka, E., Skliarov, S. (2017). Examining the learning fire detectors under real conditions of application. Eastern-European Journal of Enterprise Technologies, 3 (9 (87)), 53-59. doi: http://doi.org/10.15587/1729-4061.2017.101985

[20] Pospelov, B., Andronov, V., Rybka, E., Skliarov, S. (2017). Research into dynamics of setting the threshold and a probability of ignition detection by selfadjusting fire detectors. Eastern-European Journal of Enterprise Technologies, 5 (9 (89)), $43-48$. doi: http://doi.org/10.15587/1729-4061.2017.110092

[21] Pospelov, B., Rybka, E., Meleshchenko, R., Gornostal, S., Shcherbak, S. (2017). Results of experimental research into correlations between hazardous factors of ignition of materials in premises. Eastern-European Journal of Enterprise Technologies, 6 (10 (90)), 50-56. doi: http://doi.org/10.15587/1729-4061.2017.117789

[22] Bendat, J. S., Piersol, A. G. (2010). Random data: analysis and measurement procedures. John Wiley \& Sons. doi: http:// doi.org/10.1002/9781118032428

[23] Shafi, I., Ahmad, J., Shah, S. I., Kashif, F. M. (2009). Techniques to Obtain Good Resolution and Concentrated Time-Frequency Distributions: A Review. EURASIP Journal on Advances in Signal Processing, 2009 (1). doi: http://doi.org/10.1155/2009/673539

[24] Singh, P. (2016). Time-frequency analysis via the fourier representation. HAL, 1-7.

[25] Pretrel, H., Querre, P., Forestier, M. (2005). Experimental Study Of Burning Rate Behaviour In Confined And Ventilated Fire Compartments. Fire Safety Science, 8, 1217-1228. doi: http://doi.org/10.3801/iafss.fss.8-1217

[26] Pospelov, B., Andronov, V., Rybka, E., Popov, V., Romin, A. (2018). Experimental study of the fluctuations of gas medium parameters as early signs of fire. Eastern-European Journal of Enterprise Technologies, 1 (10 (91)), 50-55. doi: http:// doi.org/10.15587/1729-4061.2018.122419

[27] Stankovic, L., Dakovic, M., Thayaparan, T. (2014). Time-frequency signal analysis. Kindle edition, Amazon, 655.

[28] Avargel, Y., Cohen, I. (2010). Modeling and Identification of Nonlinear Systems in the Short-Time Fourier Transform Domain. IEEE Transactions on Signal Processing, 58 (1), 291-304. doi: http://doi.org/10.1109/tsp.2009.2028978

[29] Giv, H. H. (2013). Directional short-time Fourier transform. Journal of Mathematical Analysis and Applications, 399 (1), 100-107. doi: http://doi.org/10.1016/j.jmaa.2012.09.053

[30] Pospelov, B., Andronov, V., Rybka, E., Popov, V., Semkiv, O. (2018). Development of the method of frequencytemporal representation of fluctuations of gaseous medium parameters at fire. Eastern-European Journal of Enterprise Technologies, 2 (10 (92)), 44-49. doi: http://doi.org/10.15587/1729-4061.2018.125926

[31] Mandelbrot, B. (2002). Fraktalnaya geometriya prirodyi. Institut kompyuternyih issledovaniy, 656, 12.

[32] Marwan, N. (2011). How to avoid potential pitfalls in recurrence plot based data analysis. International Journal of Bifurcation and Chaos, 21 (4), 1003-1017. doi: http://doi.org/10.1142/s0218127411029008

[33] Pospelov, B. B., Andronov, V. A., Rybka, E. A., Meleschenko, R. G., Karpec, K. M. (2018). Razrabotka konstruktivnykh metodov povysheniia effektivnosti avtomaticheskogo obnaruzheniia vozgoranii v kompressornykh stanciiakh gazotransportnykh sistem Ukrainy. Kharkiv: NUGZU, 172. 